\title{
Pulse propagation, population transfer, and light storage in five-level media
}

\author{
G. Grigoryan, ${ }^{1,2}$ V. Chaltykyan, ${ }^{1,2,{ }^{*}}$ E. Gazazyan, ${ }^{1}$ O. Tikhova, ${ }^{1}$ and V. Paturyan ${ }^{3,4, \dagger}$ \\ ${ }^{1}$ Institute for Physical Research, Armenian National Academy of Sciences, Ashtarak-2 0203, Armenia \\ ${ }^{2}$ Russian-Armenian (Slavonic) University, Yerevan-51 0051, Armenia \\ ${ }^{3}$ Department of Computer Science, Maynooth University, Maynooth, County Kildare, Ireland \\ ${ }^{4}$ School of Theoretical Physics, Dublin Institute for Advanced Studies, 10 Burlington Road, Dublin 4, Ireland
}

(Received 5 November 2014; published 2 February 2015)

\begin{abstract}
We consider adiabatic interaction of five-level atomic systems and their media with four short laser pulses under the condition of all two-photon detunings being zero. We derive analytical expressions for eigenvalues of the system's Hamiltonian and determine conditions of adiabaticity for both the atom and the medium. We analyze, in detail, the system's behavior when the eigenvalue with nonvanishing energy is realized. As distinct from the usual dark state of a five-level system (corresponding to zero eigenvalue), which is a superposition of three states, in our case the superposition of four states does work. We demonstrate that this seemingly unfavorable case nevertheless completely imitates a three-level system not only for a single atom but also in the medium, since the propagation equations are also split into those for three- and two-level media separately. We show that, under certain conditions, all the coherent effects observed in three-level media, such as population transfer, light slowing, light storage, and so on, may efficiently be realized in five-level media. This has an important advantage that the light storage can be performed twice in the same medium; i.e., the second pulse can be stored without retrieving the first one, and then the two pulses can be retrieved in any desired sequence.
\end{abstract}

DOI: 10.1103/PhysRevA.91.023802

PACS number(s): 42.50.Gy, 42.65.Tg, 32.80.Qk

\section{INTRODUCTION}

Coherent interactions of light signals with quantum systems have attracted considerable interest for their importance in both fundamental science and practical applications. A prominent example of coherent interactions is electromagnetically induced transparency (EIT) [1-3], which can be used to eliminate the resonant absorption of a laser beam incident upon a coherently driven medium with appropriate energy levels. EIT technique allows controlled manipulations of the optical properties of atomic or atomlike media via coupling them with signal and control fields. In particular, it is possible to greatly slow down the optical (signal) pulse [4-6] and even stop it to attain reversible storage and retrieval of information [7-9].

Despite a huge number of publications, light storage remains in the focus of attention of researchers, since it is one of the key components in optical (quantum) information processing [1,10-14]. Another application of coherent interactions is controllable population transfer between the atomic levels and constructing desired coherent superpositions of different states [15-17]. These effects are also employed widely in such fields of research as laser cooling of atoms, lasing without inversion, new precision techniques of magnetometry, coherent control of chemical reactions, and so on.

All the above-listed phenomena have been comprehensively studied, both theoretically and experimentally, for various three-level systems and their media [18-24]. Although multilevel atomic and atomlike systems do not provide new physical principles in addition to quantum interference and the principle of superposition, they widen essentially the possibilities of experimental realizations and practical applications.

\footnotetext{
* Deceased.

†misha@cs.nuim.ie
}

The idea of a double-EIT (DEIT) regime is introduced in Ref. [25] and modified in Ref. [26]. The laser cooling scheme for trapped atoms or ions which is based on DEIT is discussed in Ref. [27]. DEIT in a medium, consisting of four-level atoms in the inverted-Y configuration, is discussed in Ref. [28]. DEIT in a ring cavity is studied in Ref. [29]. Enhanced cross-phase modulation based on DEIT is reported in Ref. [30]. Zimmer et al. [31] examine dark-state polariton formation in a four-level system. Quantum memory for light via stimulated off-resonant Raman process is considered in Ref. [32] beyond the three-level approximation. Choi and Elliott [33] propose, through numerical calculations, to use multilevel systems involving hyperfine structure in problems of localization of excitations via dark-state formation in EIT processes. Ottaviani et al. [34] investigate five-level atoms and media driven by four light pulses in a nonadiabatic regime. Two of four pulses are assumed to be weak and treated as perturbation in the first order. Chen et al. [35] observed experimentally offresonance EIT-based group delay in multilevel $D_{2}$ transitions in rubidium. Enhancement of EIT in a double- $\Lambda$ system in cesium atomic vapor by specific choice of atomic velocity distribution is observed in Ref. [36]. A scheme based on two sequential Stimulated Raman Adiabatic Passage (STIRAP) processes with four laser fields is proposed in Ref. [37] for measurement of a qubit of two magnetic sublevels of the ground state of alkaline-earth metal ions. Another topic where multilevel systems were used was generalization of the notion of dark-state polariton [38] and discussion of the possibility of applying multilevel EIT to quantum information processing.

In the present article we study both analytically and numerically a five-level atomic system interacting adiabatically with four copropagating laser pulses of different durations and different sequences of turning on and off. We require that each laser pulse interacts (be resonant) with only one of the adjacent transitions and assume all the two-photon detunings to be zero. Two examples of such level diagrams are shown in 
(a)

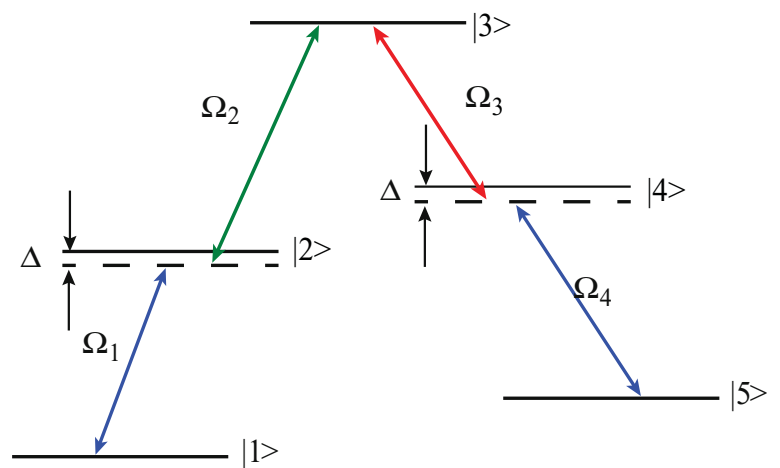

(b)

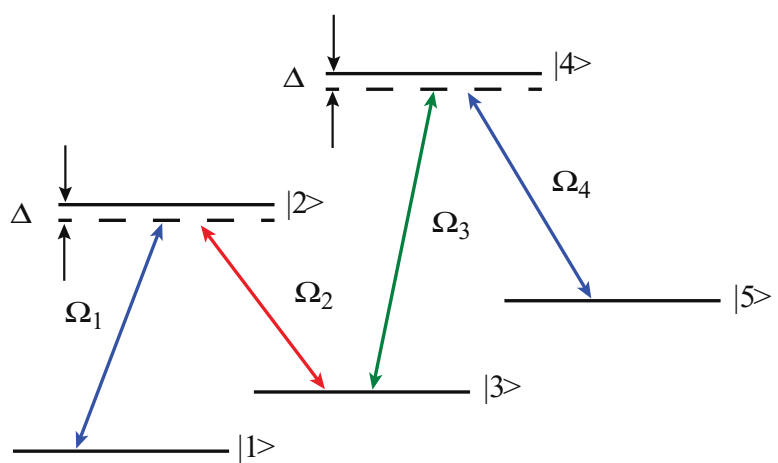

FIG. 1. (Color online) Five-level coupling schemes: (a) extended $\Lambda$ scheme and (b) M-type scheme.

Fig. 1. Another example is the ladder system, which may turn out to be rather useful for problems of excitation of Rydberg states.

As distinct from all the above-cited works, we concentrate on those eigenstates of an interaction Hamiltonian (see below) whose eigenvalues are different from zero. We show that these eigenstates are similar to the well-known dark and bright states in a three-level system. As distinct from the state in the $\mathrm{M}$ system considered in Ref. [15], the levels 2 and 4 are at interaction with laser fields populated, but the population of level 3 remains zero. We demonstrate for our case that an efficient and more flexibly controllable population transfer and light storage becomes possible. We also study the advantages of this technique. Specifically, we show the possibility of successive storage of two pulses with their subsequent retrieval. The first pulse is stored in the coherence $\rho_{51}$, which exactly reproduces, after turning off the interaction, the shape of the pulse $\Omega_{2}$. Since the coherence $\rho_{31}$ remains zero for the duration of the interaction, the same medium can be used again for storage of another pulse.

The article is organized as follows. In Sec. II we derive eigenfunctions and eigenvalues of systems under consideration and discuss the relevant cases. In Sec. III we study adiabatic population transfer in five-level systems. Section IV derives the equations of propagation and presents their analytical solution. In the same section the regime of adiabaton is demonstrated. In Sec. V we show the possibility to store optical information in considered media. We conclude with a final discussion in Sec. VI.

\section{EIGENFUNCTIONS AND EIGENVALUES OF INTERACTION HAMILTONIAN}

Consider a five-level atomic system as shown in Fig. 1. Four (in general) laser pulses are close to resonance with respective transitions (Fig. 1). The Hamiltonian of interaction in the rotating-wave approximation, and under the assumptions that the carrier frequencies of laser pulses are tuned near resonance with one of the adjacent atomic transitions and that pulse durations are much shorter compared to relaxation times in the system, has the following form:

$$
H=\sum_{i} \sigma_{i, i} \delta_{i-1}-\left(\sum_{i} \sigma_{i, i+1} \Omega_{i}+\text { H.c. }\right),
$$

with the projection matrices $\sigma_{i j}=|i\rangle\langle j|$, the Rabi frequencies $\Omega_{i}$ at transitions $i \rightarrow i+1$, and $\delta_{i-1}$ representing $(i-1)$ photon detunings (with $\delta_{0}=0$ ). The Rabi frequencies are assumed to be real and positive. Phases, which can vary during propagation, are included in the single-photon detunings $\left(\Delta_{i}=\omega_{i+1, i}-\omega_{i}+\dot{\varphi}_{i}\right.$, if $\omega_{i+1, i}>0$, and $\Delta_{i}=\omega_{i, i+1}-$ $\omega_{i}+\dot{\varphi}_{l}$ if $\left.\omega_{i+1, i}<0\right)$. The definition of multiphoton detunings depends on the specific scheme of interaction. For an $\mathrm{M}$ system [see Fig. 1(b)] the multiphoton detunings are $\delta_{2}=\Delta_{1}-\Delta_{2}$, $\delta_{3}=\Delta_{3}+\delta_{2}$, and $\delta_{4}=\Delta_{4}-\delta_{3}$. For an extended $\Lambda$ system [see Fig. 1(a)], the multiphoton detunings are $\delta_{2}=\Delta_{1}+\Delta_{2}$, $\delta_{3}=-\Delta_{3}+\delta_{2}$, and $\delta_{4}=-\Delta_{4}+\delta_{3}$.

Eigenvalues of the Hamiltonian (1) can easily be derived analytically if all of the two-photon detunings are zero, i.e.,

$$
\delta_{2}=0, \quad \delta_{3}-\delta_{1}=0, \quad \delta_{4}-\delta_{2}=0 .
$$

For an $\mathrm{M}$ system, these conditions mean equal single-photon detunings, while for the extended $\Lambda$ system the single-photon detunigs have equal absolute values, but differ in sign (see Fig. 1). When conditions (2) are met, one of five eigenvalues of the Hamiltonian is $\lambda=0$. The detailed calculations of the remaining four eigenvalues are presented in the Appendix.

Consider now a special case, when the pulses $\Omega_{1}$ and $\Omega_{4}$ coincide by their temporal profiles (but the frequencies and phases of pulses may be different). In this case, the eigenvalues of the Hamiltonian (1) are

$$
\begin{aligned}
\lambda_{0} & =0 \\
\lambda_{1,3} & =\frac{1}{2}\left(\Delta \mp \sqrt{\Delta^{2}+4 \Omega_{1}^{2}}\right), \\
\lambda_{2,4} & =\frac{1}{2}\left(\Delta \mp \sqrt{\Delta^{2}+4\left(\Omega_{1}^{2}+\Omega_{2}^{2}+\Omega_{3}^{2}\right)}\right) .
\end{aligned}
$$

We note that, when the fields are turned off, we get $\lambda_{1,2} \rightarrow 0$ and $\lambda_{3,4} \rightarrow \Delta$. It should be emphasized that the eigenvalues $\lambda_{1,3}$ depend upon only the field $\Omega_{1}$ and coincide with the eigenvalues of a two-level system, driven by field $\Omega_{1}$. Similarly, the eigenvalues $\lambda_{2,4}$ are equal to the eigenvalues of a twolevel system, driven by an effective field $\left(\Omega_{1}^{2}+\Omega_{2}^{2}+\Omega_{3}^{2}\right)^{1 / 2}$. Adiabatic evolution requires the following conditions to be met (see the Appendix for details):

$$
\Delta T \gg 1, \quad \frac{\left(\Omega_{2}^{2}+\Omega_{3}^{2}\right) T}{\Delta} \gg 1, \quad \frac{\Omega_{1}^{2} T}{\Delta} \gg 1,
$$

with the duration $T$ of the shortest pulse. The first condition mirrors the adiabaticity condition for a two-level system. The second condition corresponds to the adiabaticity condition 
for a three-level system. The third condition is only relevant in the time interval where all pulses overlap (i.e., when $\Omega_{2}^{2}+\Omega_{3}^{2} \neq 0$ ).

To write the eigenvectors corresponding to the eigenvalues $\lambda_{1}$ and $\lambda_{2}$ we introduce the following notations:

$$
\begin{aligned}
\Omega^{2} & =\Omega_{2}^{2}+\Omega_{3}^{2}, \quad \tan \theta=\frac{\Omega_{2}}{\Omega_{3}}, \\
\tan \Phi_{1} & =-\frac{\lambda_{1}}{\Omega_{1}}, \quad \tan \Phi_{2}=-\frac{\lambda_{2}}{\Omega_{1}}, \\
\tan \Phi & =-\frac{\Omega}{\Omega_{1}} \cos \Phi_{2} .
\end{aligned}
$$

Then, the eigenvector corresponding to the eigenvalue $\lambda_{1}$ is

$$
\left|\lambda_{1}\right\rangle=\left|\psi_{1}\right\rangle \cos \theta-\left|\psi_{2}\right\rangle \sin \theta
$$

where $\left|\psi_{1}\right\rangle$ and $\left|\psi_{2}\right\rangle$ are superposition states of the two-level systems $1 \rightarrow 2$ and $5 \rightarrow 4$ :

$$
\begin{aligned}
& \left|\psi_{1}\right\rangle=\cos \phi_{1}|1\rangle-\sin \phi_{1}|2\rangle, \\
& \left|\psi_{2}\right\rangle=\cos \phi_{1}|5\rangle-\sin \phi_{1}|4\rangle .
\end{aligned}
$$

It is apparent that the eigenvector corresponding to $\lambda_{1}$ does not involve state $|3\rangle$ and is equal to the dark state of a three-level $\Lambda$ system, if we replace the lower states by the superposition states $\left|\psi_{1}\right\rangle$ and $\left|\psi_{2}\right\rangle$.

Similarly, the eigenvector corresponding to the eigenvalue $\lambda_{2}$ yields

$$
\left|\lambda_{2}\right\rangle=\left|\psi_{1}^{\prime}\right\rangle \cos \Phi \sin \theta-\sin \phi|3\rangle+\left|\psi_{2}^{\prime}\right\rangle \cos \Phi \cos \theta,
$$

where

$$
\begin{gathered}
\left|\psi_{1}^{\prime}\right\rangle=\cos \Phi_{2}|1\rangle-\sin \Phi_{2}|2\rangle, \\
\left|\psi_{2}^{\prime}\right\rangle=\cos \Phi_{2}|5\rangle-\sin \Phi_{2}|4\rangle
\end{gathered}
$$

As in the previous case, the eigenvector $\left|\lambda_{2}\right\rangle$ is equal to that of the bright state of a three-level $\Lambda$ system $[39,40]$, if we replace the lower states by the superposition states $\left|\psi_{1}^{\prime}\right\rangle$ and $\left|\psi_{2}^{\prime}\right\rangle$. The time behavior of eigenvalues $\lambda_{i}$ in the special case above for different pulse sequences is demonstrated in Figs. 2 and 3.

\section{POPULATION TRANSFER}

As follows from the expressions (6) and (8) the five-level system imitates the three-level $\Lambda$ system. Thus, we can use the state $\left|\lambda_{1}\right\rangle$ to transfer the system from state $|1\rangle$ to state $|5\rangle$ by a STIRAP-like process, driven by the pulse sequence introduced above (see Fig. 4). In contrast to a simple three-level $\Lambda$ system, during the interaction some transient population shows up in the intermediate levels $|2\rangle$ and $|4\rangle$ of the fivelevel system. However, these transient populations are very small, if the one-photon detuning is sufficiently large, but still satisfies the adiabaticity condition (3). It should be noted that the condition of large one-photon detuning is not very crucial for the population transfer. The dynamics of populations in the described case is shown in Fig. 4.

Similarly, the state $\left|\lambda_{2}\right\rangle$ is analogous to the bright state of a $\Lambda$ system and we can use these states for adiabatic transfer from state $|5\rangle$ to state $|1\rangle$ by a b-STIRAP-like process $[39,40]$ driven by the pulse sequence in Fig. 3 , because the state $\left|\lambda_{2}\right\rangle$
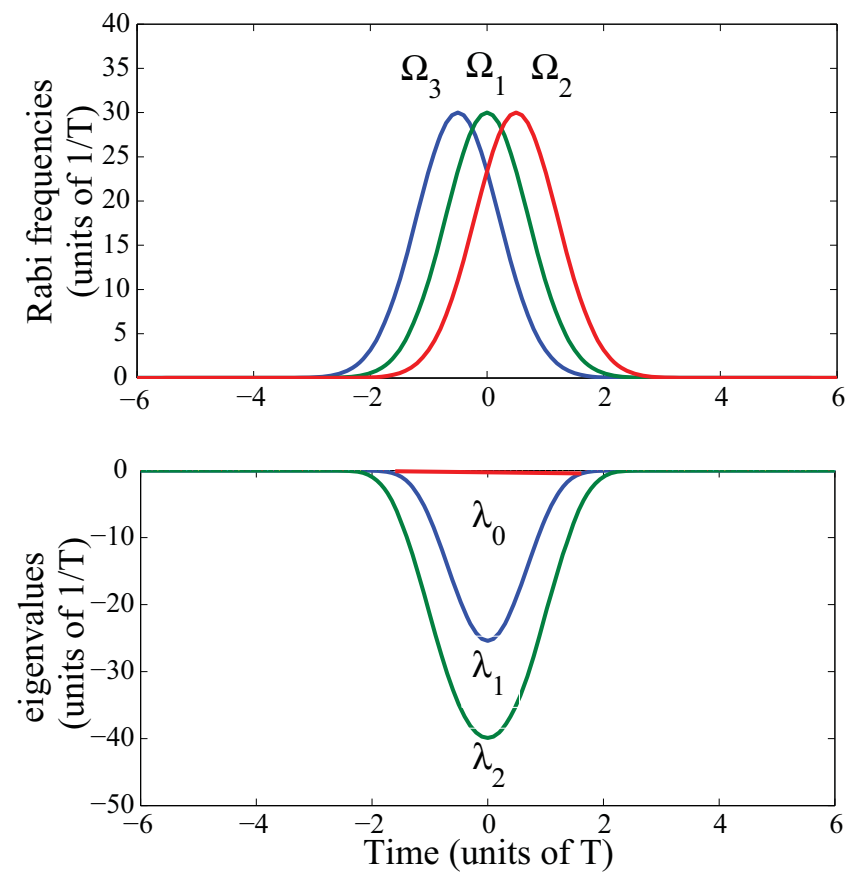

FIG. 2. (Color online) Time dependencies of the pulses (top) and the adiabatic energies (bottom) for considered systems. The shapes of all pulses are Gaussian. The single-photon detuning is $\Delta=10 / T$. It is seen clearly that in the range of overlapping of the pulses, the adiabaticity of interaction is ensured.

is not realized with the pulse sequence of Fig. 2, according to the definition of the angle $\Phi$. We emphasize that the STIRAP technique is applicable for both schemes of pulse sequence in Figs. 2 and 3. The dynamics of populations in the
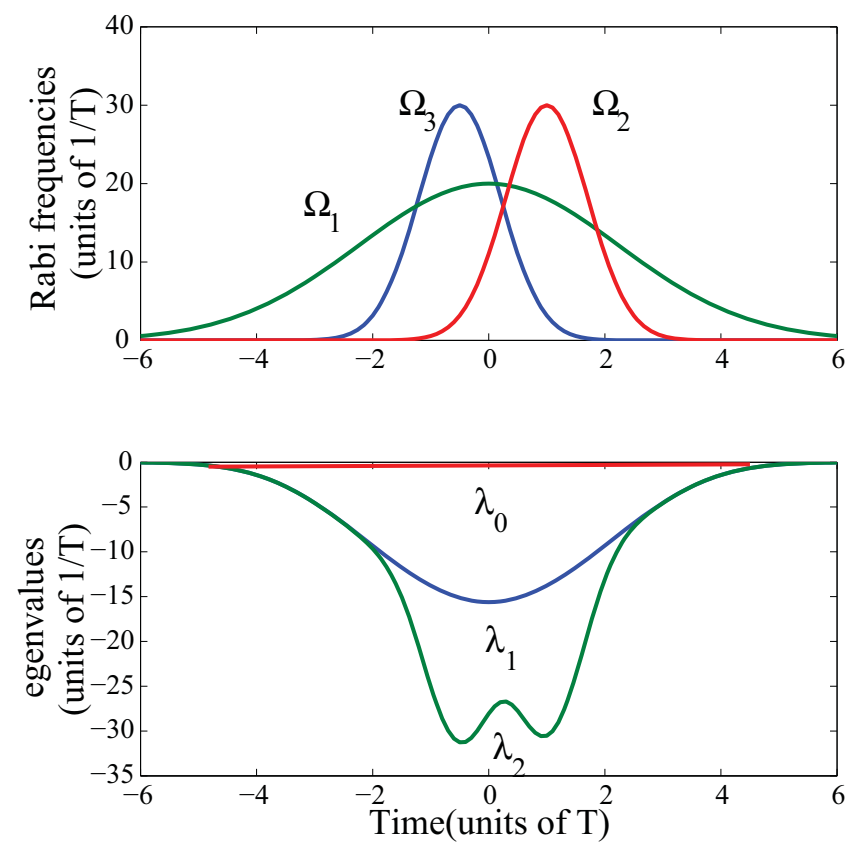

FIG. 3. (Color online) Same as in Fig. 2, but for another sequence of pulses. 

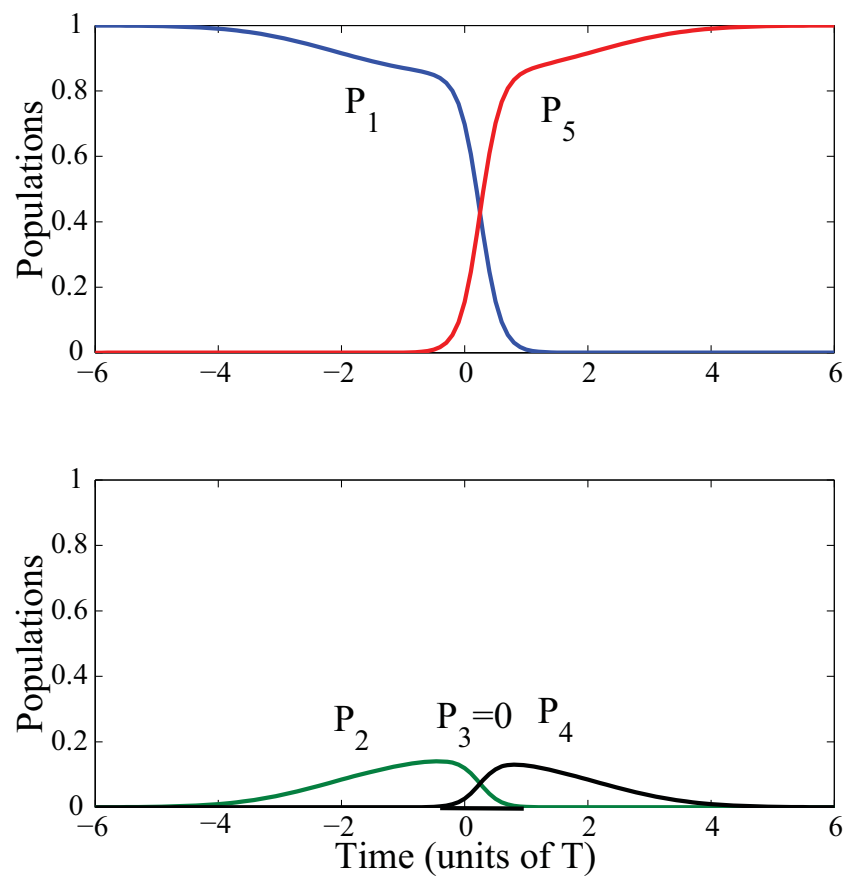

FIG. 4. (Color online) Dynamics of the population transfer from the initial state $|1\rangle$ to the final state $|5\rangle$ if the atom is in state $\left|\lambda_{1}\right\rangle$ and the pulse sequence is the same as that in Fig. $3\left(P_{i}=\left|\left\langle i \mid \lambda_{1}\right\rangle\right|^{2}\right)$. The single photon detuning is $\Delta=20 / T$.

b-STIRAP case are demonstrated in Fig. 5. Note that the two eigenstates $\left|\lambda_{1}\right\rangle$ and $\left|\lambda_{2}\right\rangle$ render the five-level system, driven by a considered pulse sequence, fully reversible. Thus, we can transfer atomic population from state $|1\rangle$ to state $|5\rangle$ by a STIRAP-like process and from state $|5\rangle$ to state $|1\rangle$ by a b-STIRAP-like process with the same sequence of pulses.

\section{MEDIUM OF ATOMS}

Now we move from a single-atom case to that of a medium consisting of the described atoms. We start from the wellknown truncated Maxwell equation in running coordinates $x$, $\tau=t-x / c .:$

$$
\frac{\partial E_{i}}{\partial x}=i \frac{2 \pi \omega_{i}}{c} N d_{i}
$$

Here $E_{i}$ are the complex amplitudes of electric fields of the pulses, $N$ is the number density of medium atoms, and $d_{i}$ are the amplitudes of induced dipole moments of each individual atom at a frequency $\omega_{i},\langle\psi|d| \psi\rangle=\sum d_{i} \exp \left(-i \omega_{i} t\right)+$ c.c. These amplitudes can be expressed in terms of the amplitudes of atomic populations $b_{i}$ of bare states and the matrix elements of the dipole moment $\langle i|d| i+1\rangle: d_{i}=b_{i}^{*} b_{i+1}\langle i|d| i+1\rangle$ if $\omega_{i+1, i}>0$ and $d_{i}=b_{i} b_{i+1}^{*}\langle i|d| i+1\rangle$ if $\omega_{i+1, i}<0$. The coefficients $b_{i}$ are determined by the nonstationary Schrödinger equation with the Hamiltonian (1).

Separating real and imaginary parts in the truncated equation of propagation, differentiating the equation for the phase with respect to time, and combining the obtained equations with the Schrödinger equation, we obtain in the general case a self-consistent system of equations describing the variation
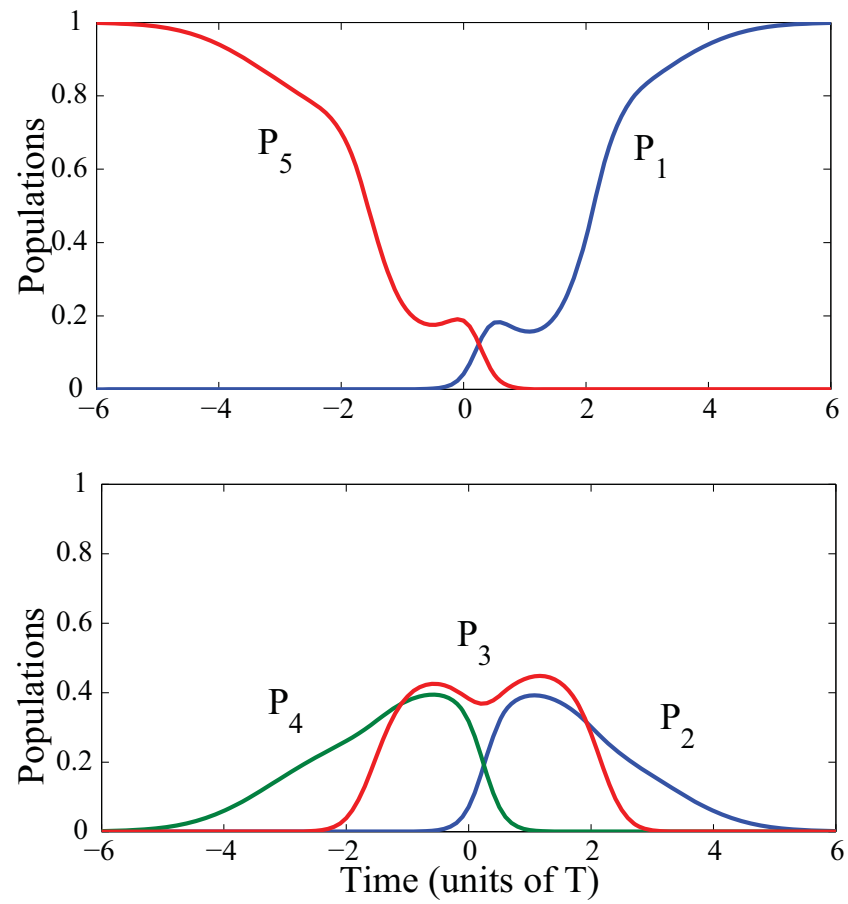

FIG. 5. (Color online) Dynamics of the population transfer from the initial state $|5\rangle$ to the final state $|1\rangle$ if the atom is in state $\left|\lambda_{2}\right\rangle$. The pulse sequence and parameters are the same as those in Fig. 4 $\left(P_{i}=\left|\left\langle i \mid \lambda_{2}\right\rangle\right|^{2}\right)$.

of frequencies (one-photon detunings) and intensities (Rabi frequencies) of pulses during propagation in medium. For example, in the case of the medium consisting of M-type atoms we obtain

$$
\begin{aligned}
& \frac{\partial \Omega_{1}^{2}}{\partial x}=q_{1} \frac{\partial\left|b_{1}\right|^{2}}{\partial \tau}, \\
& \frac{\partial \Omega_{2}^{2}}{\partial x}=-q_{2} \frac{\partial\left(\left|b_{1}\right|^{2}+\left|b_{2}\right|^{2}\right)}{\partial \tau}, \\
& \frac{\partial \Omega_{3}^{2}}{\partial x}=-q_{3} \frac{\partial\left(\left|b_{4}\right|^{2}+\left|b_{5}\right|^{2} \mid\right)}{\partial \tau}, \\
& \frac{\partial \Omega_{4}^{2}}{\partial x}=q_{5} \frac{\partial\left|b_{5}\right|^{2}}{\partial \tau}, \\
& \frac{\partial \Delta_{i}}{\partial x}=q_{i} \frac{\partial}{\partial \tau} \frac{\operatorname{Re}\left(b_{i}^{*} b_{i+1}\right)}{\Omega_{i}},
\end{aligned}
$$

where $q_{i}=2 \pi N \omega_{i}\left|d_{i, i+1}\right|^{2} / c \hbar$ is the propagation constant. In the case of an extended $\Lambda$ system [Fig. 1(a)] the equations remain essentially the same, but we must change the signs of the right-hand sides in the second and third equations. As follows from Eqs. (11), during propagation in the medium not only the shapes of pulses may vary essentially but also the conditions for detuning of resonances may be violated. Variations of resonance detunings are caused by processes of self-phase modulation (parametric broadening of the pulse spectrum [41]). The modification of the shapes of pulses is caused both by the nonlinear group velocity (which can result in formation of shock wave fronts [42]) and by energy transfer between the pulses (which can lead to full depletion of one of 
the pulses [43]). It is, however, obvious that all these processes are proportional to the length of propagation. Hence, if the optical length of the medium is sufficiently short, the variations of detunings and intensities can be negligibly small.

Since it is only the time derivatives that enter the right-hand sides of Eqs. (11), we can use expressions (6) and (8) for the atomic amplitudes in these equations and this will be equivalent to allowance for the first nonadiabatic corrections. Correspondingly, the conditions of smallness of the right-hand sides of Eqs. (11) serve as a criteria of the insignificance of changes in spatial and temporal characteristics of pulses and thus a criteria of adiabaticity of the interaction in the medium. For simplicity we restrict ourselves to the case of equal oscillator strengths in all transitions (in the case of different oscillator strengths, we can proceed as in the three-level system [44]). In case of the state $\left|\lambda_{1}\right\rangle$ we now obtain from Eq. (11)

$$
\begin{aligned}
\frac{\partial\left(\Omega_{1}^{2}+\Omega_{4}^{2}\right)}{\partial x} & =q \frac{\partial \cos ^{2} \Phi_{1}}{\partial \tau}, \\
\frac{\partial\left(\Delta_{1}+\Delta_{4}\right)}{\partial x} & =-q \frac{\partial}{\partial \tau} \frac{\cos \left(2 \Phi_{1}\right)}{\Delta}, \\
\frac{\partial \Delta_{2}}{\partial x} & =-\frac{\partial \Delta_{3}}{\partial x}=0 \\
\frac{\partial \Omega^{2}}{\partial x} & =0 \\
\frac{\partial \theta}{\partial x}+\frac{q}{\Omega^{2}} \frac{\partial \theta}{\partial \tau} & =0 .
\end{aligned}
$$

We emphasize that the system of Eqs. (12) has an interesting and important peculiarity. Propagation of fields $\Omega_{2}$ and $\Omega_{3}$ occurs independent of fields $\Omega_{1}$ and $\Omega_{4}$ and is described by propagation equations for a three-level-atom medium under conditions of dark-state formation [1]. Fields $\Omega_{1}$ and $\Omega_{4}$ are described by propagation equations for a two-level-atom medium [42]. This peculiarity is important because both problems are studied in sufficient detail in the literature and have analytical solutions. In particular, we can realize all phenomena taking place in the usual $\Lambda$ systems with the three-level 2-3-4 system which is supported by two-level 1-2 and 4-5 systems pumping level 2 and depleting level 4, respectively. As an example, we obtain, in the considered five-level system, propagation of the adiabaton [45] in the five-level system. Figure 6 visualizes this phenomenon (details are in the figure caption).

Equations (12) are valid if the state $\left|\lambda_{1}\right\rangle$ is formed on the entire length of the medium. This requires fulfillment of two conditions: (i) the detunings $\left|\Delta_{i}\right|=\Delta$ for all $i$ and Rabi frequencies $\Omega_{1}=\Omega_{4}$ and (ii) the adiabaticity of interaction in all of the medium. Let us examine when these conditions are met. Equations (12) show that detunings $\Delta_{2}$ and $\Delta_{3}$ are preserved during propagation (as they should be in a three-level system), whereas $\Delta_{1}$ and $\Delta_{4}$ can vary with propagation length because of self-phase modulation (as in a two-level system), but, as shown in Ref. [42], these variations can be neglected if we limit the length by

$$
\frac{q x}{\Delta} \frac{1}{\Delta T} \ll 1 .
$$
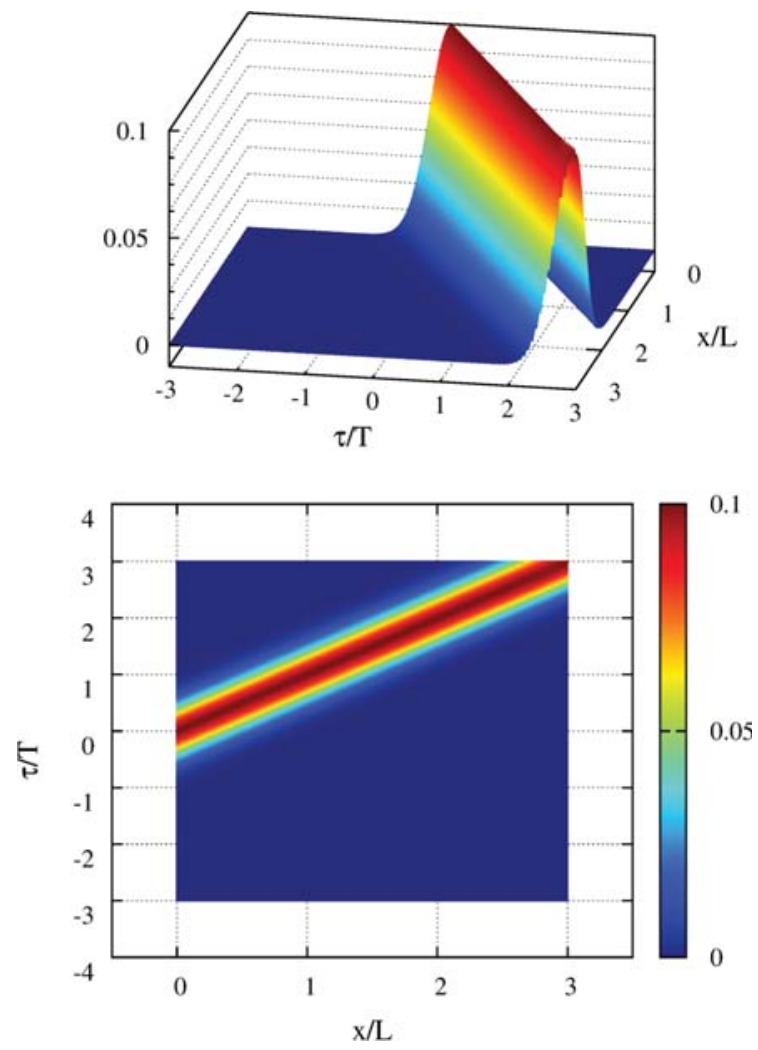

FIG. 6. (Color online) Distortion-free propagation of the signal pulse $\Omega_{2}$ at the subluminal group velocity. Shapes of pulses are chosen to be $\Omega_{1} T=\Omega_{4} T=\Omega_{3} T=30 e^{\left(-0.2(\tau / T)^{2}\right)}$ and $\Omega_{2} T=0.1 e^{\left(-5(\tau / T)^{2}\right)}$ (linear case). The single-photon detuning is $\Delta=100 / T$. The scaled length $L=\Omega_{0}^{2}(\tau=0) T / q$ and the time delay in the medium is $\Delta t / T=x / L$.

On the same length we can take $\Omega_{1}=\Omega_{4}$ (adiabatic approximation for a two-level system). As follows from the results of cited works the adiabaticity of interaction in a two-level system breaks at the lengths when $\left(q x / \Delta^{2} T\right) \sim 1$, whereas the interaction adiabaticity in a three-level medium does not break at all. Another condition imposed on the length requires nondepletion of the pump pulse in a three-level medium for an effective population transfer [43]:

$$
\frac{q x}{\Delta} \frac{\Delta}{\Omega^{2} T} \sim 1 \text {. }
$$

It follows from Eqs. (13) and (14) that the influence of the medium is determined by the factor $q x / \Delta$ times the adiabaticity conditions for a single atom. This means that it is sufficient to require the medium parameter $q x / \Delta$ to not exceed unity by much. If we express this parameter in terms of the linear coefficient of absorption of medium $\alpha_{0}$, we obtain restriction for the optical length in the form

$$
\frac{q x}{\Delta}=\alpha_{0} x \frac{\Gamma}{\Delta} \sim 1,
$$

with $\Gamma$ being the maximum of the relevant widths.

So, in the case of a large one-photon detuning the length of the adiabaticity of interaction can exceed the length of linear absorption in the medium several times. On this length 

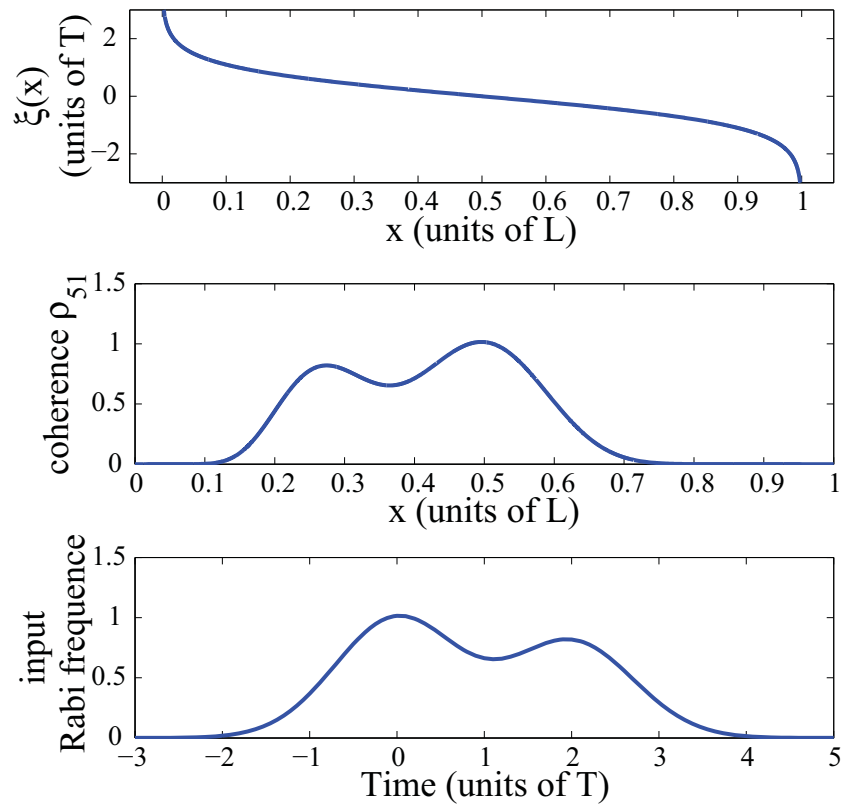

FIG. 7. (Color online) The $x$ dependence of the $\xi(x)$ function and the spatial distribution of coherence $\rho_{51}$ after the interaction is switched off (top) and the temporal profile of the pulse $\Omega_{2}$ at the medium input (bottom).

Eqs. (12) can be solved analytically and the solution has the following form:

$$
\begin{aligned}
& \Omega_{1}=\Omega_{4}=\Omega_{10}(\tau), \\
& \Omega_{2}=\Omega_{0}(\tau) \sin \theta_{0}(\xi(x, t)), \\
& \Omega_{3}=\Omega_{0}(\tau) \cos \theta_{0}(\xi(x, t)),
\end{aligned}
$$

where $\Omega_{10}, \Omega_{0}$, and $\theta_{0}$ are the boundary conditions given at the entrance of the medium and $\xi(x, t)$ is an implicit function defined by the following expression:

$$
\int_{\xi}^{\tau} \Omega_{0}^{2} d t=q x
$$

We note that all of the above is true only if the dressed state $\left|\lambda_{1}\right\rangle$ is realized in the medium. In case then, as a result of an adiabatic interaction, the other dressed state (for example $\left|\lambda_{2}\right\rangle$ ) is realized, the propagation equations (11) are no longer split, and finding their solution requires additional investigation.

\section{LIGHT STORAGE}

It follows from the solution (16) that, after turning off all pulses, the coherence $\rho_{15}$ induced by these pulses remains in the medium (like in the three-level system):

$$
\rho_{15}=-\sin \theta(\xi) \cos \theta(\xi),
$$

where function $\xi(x)$ is defined by the following expression:

$$
\int_{\xi}^{\infty} \Omega_{0}^{2} d t=q x
$$

Figure 7 shows $x$ dependence of the $\xi$ function and coherence $\rho_{15}$ after all pulses are turned off, together with the input shape of the probe pulse. The figure demonstrates
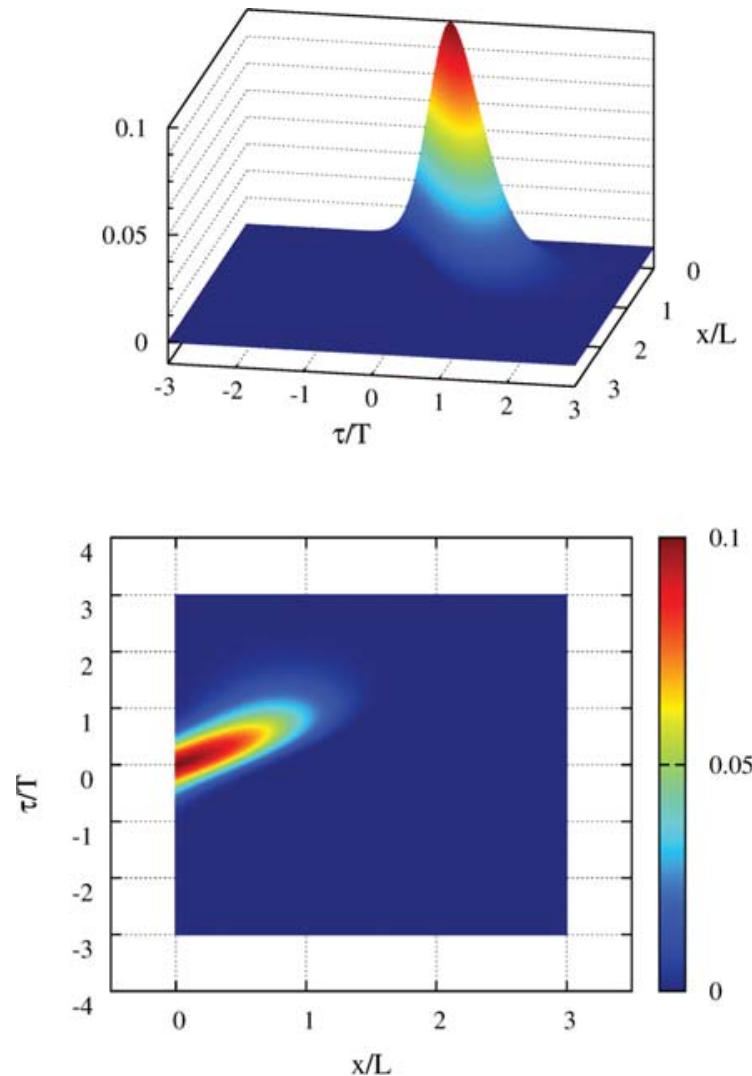

FIG. 8. (Color online) Propagation of the signal pulse $\Omega_{2}$ (light storage). Shapes of pulses are chosen to be $\Omega_{1} T=\Omega_{4} T=$ $30 e^{\left(-3(\tau / T)^{2}\right)}, \quad \Omega_{3} T=30 e^{\left(-(\tau / T)^{2}\right)}, \quad$ and $\Omega_{2} T=0.1 e^{\left(-5(\tau / T)^{2}\right)}$. The single-photon detuning is $\Delta=100 / T$. The group velocity $u=$ $c /\left(1+q c / \Omega_{3}^{2}\right) \rightarrow 0$ when $\Omega_{3}^{2} \rightarrow 0$.

that the distribution of coherence along $x$ mirrors the arbitrary $t$-shape of the probe pulse at the medium entrance. It is also apparent that the $\xi$ function has two asymptotes, $x=0$ and $x=x_{\max }$. The existence of the maximal length of the medium, i.e., the length where the probe pulse disappears, is the essence of the light storage phenomenon. For the realization of storage (and the mapping of the $t$ dependence onto the $x$ distribution) the medium must not be shorter than $x_{\max }$. It follows from Eq. (19) that the maximal length is representable in the form $x_{\max } N=N_{\mathrm{ph}}$, where $N$ is the number density of resonant atoms in the medium and $N_{\mathrm{ph}}$ is the overall photon fluxes in control and probe pulses at the medium input:

$$
N_{\mathrm{ph}}=\int_{-\infty}^{\infty}\left(c E_{p 0}^{2} / \hbar \omega_{p}+c E_{s 0}^{2} / \hbar \omega_{s}\right) d t
$$

Thus, in order to write completely a light pulse into a medium, it is necessary that the number of atoms interacting with radiation be comparable with the total number of relevant photons. We emphasize that $x_{\max }$ does not depend on $\Omega_{1}$ and $\Omega_{4}$ (the latter enters only the adiabaticity condition). Note that in the linear approximation in $\Omega_{2}$ we can construct a dark-state polariton similar to that in a $\Lambda$ system [7].

Figure 8 shows, by means of numerical solution of corresponding equations with the use of the Lax-Wendroff method $[46,47]$, the process of writing a light pulse into 
a medium. The described process of light storage is more visualizable in the generalized $\Lambda$ system, but has no principle advantages as compared to the usual $\Lambda$ system. In contrary, the $M$ system is much more interesting because it enables double storage; i.e., we can write two different pulses, one after another with the possibility of subsequent retrieval in any desired succession. Indeed, during the whole interaction time (and also after the first writing), the coherence $\rho_{31}$ remains zero and the population of the level 1 is close to unity (in linear approximation in $\Omega_{2}$ ). This means that the same medium is ready for the usual $\Lambda$ storage of the second pulse. For example, we can write the pulse $\Omega_{1}$ into the same medium. For this purpose the pulses $\Omega_{1}$ and $\Omega_{2}$ should be divided into two beams before the first storage attempt. The weak portion of $\Omega_{1}$ and the strong portion of $\Omega_{2}$ should be sent to a delay line, to be used for the second storage (using $\Omega_{2}$ as a control pulse).

\section{CONCLUSION}

We considered the behavior of a five-level atomic system and a medium of such systems driven by four laser pulses of different amplitudes and frequencies. We showed for such a system the possibility of analytical determination of system eigenfunctions and eigenvalues in the case where all twophoton detunings are zero. We have obtained that, in addition to the traditional zero eigenvalue, there exists a nonzero one, for which the propagation equations in the medium are split into the equations for two- and three-level system media, i.e., two of the four laser pulses travel independently of the other two. This splitting is caused by the fact that in this case the five-level system reduces to a certain effective " $\Lambda$ " system whose ground states are superpositions of two states. We derive the dressed states and dressed energies of the system, as well as conditions for adiabatic evolution, and show that the length of medium where adiabaticity is preserved exceeds several times the linear absorption length. We show that adiabatic passage permits reversible transfer of the atomic population from an initial to a target state, and back again. The obtained mechanism of the population transfer may be employed for excitation of Rydberg states in atoms. We analyzed the traveling of pulses in the medium and obtained, in particular, adiabaton (distortion-free) propagation at the group velocity lower than $c$. Also the process of information storage in a five-level medium was examined. We propose a possibility of double storage of light pulses in the same medium with subsequent retrieval of the two stored pulses in the desired sequence. We note that the relaxation processes have not been taken into account throughout the work. Allowance for these processes requires separate investigation. Finally we note that the considered five-level systems can experimentally be realized in a number of media, such as hyperfine structures of D lines of alkali-metal atoms, in optical transitions of rare-earth-ion impurities in crystal matrices, in rovibrational levels of different electronic states in molecules, in problems of population transfer in entangled three two-level atoms, and so on.

\section{ACKNOWLEDGMENTS}

The research leading to these results has received funding from the Seventh Framework Programme FP7/2007-2013/ under REA Grant No. 295025. We acknowledge additional support from the IRMAS International Associated Laboratory (CNRS-France, SCS-Armenia).

\section{APPENDIX}

The equation for eigenvalues of the interaction Hamiltonian, i.e., the equation $\operatorname{det}(H-\lambda I)=0$, has, under conditions $\delta_{2}=\delta_{4}=0$ and $\delta_{1}=\delta_{3}=\Delta$, the following form:

$$
\lambda^{2}(\lambda-\Delta)\left[\lambda(\lambda-\Delta)+\Omega_{s}^{2}\right]+V^{4} \lambda=0
$$

where $\Omega_{s}^{2}=\Omega_{1}^{2}+\Omega_{2}^{2}+\Omega_{3}^{2}+\Omega_{4}^{2} \quad$ and $\quad V^{4}=\Omega_{2}^{2} \Omega_{4}^{2}+$ $\Omega_{1}^{2} \Omega_{3}^{2}+\Omega_{1}^{2} \Omega_{4}^{2}$. With the notation $x=\lambda(\lambda-\Delta)$ the equation above becomes $\lambda\left[x^{2}-\Omega_{s}^{2} x+V^{4}\right]=0$ and the eigenvalues are obtained directly:

$$
\begin{aligned}
\lambda_{0} & =0, \\
\lambda_{3,1} & =\frac{1}{2}\left[\Delta \pm\left(\Delta^{2}+4 x_{1}\right)^{1 / 2}\right], \\
\lambda_{4,2} & =\frac{1}{2}\left[\Delta \pm\left(\Delta^{2}+4 x_{2}\right)^{1 / 2}\right],
\end{aligned}
$$

where $x_{2,1}=(1 / 2)\left[\Omega_{1}^{2} \pm\left(\Omega_{s}^{4}-4 V^{4}\right)^{1 / 2}\right]$. We note that the condition $\Omega_{s}^{4} \geqslant 4 V^{4}$ is always met.

Conditions of interaction adiabaticity for a single atom, $\left|\lambda_{i}-\lambda_{j}\right| T \gg 1$ for any $i \neq j$ with $T$ being the time of interaction, leads to the following requirement imposed on the parameters of pulses:

$$
\begin{gathered}
\frac{\left(x_{2}-x_{1}\right) T}{\left(\Delta^{2}+4 x_{2}\right)^{1 / 2}} \gg 1, \\
\left(\Delta^{2}+4 x_{1}\right)^{1 / 2} T \gg 1, \\
\frac{x_{1,2} T}{\left(\Delta^{2}+4 x_{1,2}\right)^{1 / 2}} \gg 1 .
\end{gathered}
$$

Note that the last condition can be fulfilled only for $V^{4} \neq 0$, i.e., in the range of overlapping of pulses.
[1] M. Fleischhauer, A. Imamoglu, and J. P. Marangos, Rev. Mod. Phys. 77, 633 (2005).

[2] S. E. Harris, Phys. Today 50, 36 (2008).

[3] M. O. Scully, Quantum Optics (Cambridge University Press, Cambridge, UK, 1997).

[4] L. V. Hau, S. E. Harris, Z. Dutton, and C. H. Behroozi, Nature (London) 397, 594 (1999).

[5] D. Budker, D. F. Kimball, S. M. Rochester, and V. V. Yashchuk, Phys. Rev. Lett. 83, 1767 (1999).
[6] M. M. Kash, V. A. Sautenkov, A. S. Zibrov, L. Hollberg, G. R. Welch, M. D. Lukin, Y. Rostovtsev, E. S. Fry, and M. O. Scully, Phys. Rev. Lett. 82, 5229 (1999).

[7] M. Fleischhauer and M. D. Lukin, Phys. Rev. Lett. 84, 5094 (2000).

[8] C. Liu, Z. Dutton, C. H. Behroozi, and L. V. Hau, Nature (London) 409, 490 (2001).

[9] D. F. Phillips, A. Fleischhauer, A. Mair, R. L. Walsworth, and M. D. Lukin, Phys. Rev. Lett. 86, 783 (2001). 
[10] M. D. Lukin, Rev. Mod. Phys. 75, 457 (2003).

[11] M. D. Eisaman, A. André, F. Massou, M. Fleischhauer, A. S. Zibrov, and M. D. Lukin, Nature (London) 438, 837 (2005).

[12] T. Chaneliere, D. N. Matsukevich, S. D. Jenkins, S.-Y. Lan, T. A. Kennedy, and A. Kuzmich, Nature (London) 438, 833 (2005).

[13] H. Kimble, Nature (London) 453, 1023 (2008).

[14] K. Hammerer, A. S. Sørensen, and E. S. Polzik, Rev. Mod. Phys. 82, 1041 (2010).

[15] K. Bergmann, H. Theuer, and B. W. Shore, Rev. Mod. Phys. 70, 1003 (1998).

[16] P. Král, I. Thanopulos, and M. Shapiro, Rev. Mod. Phys. 79, 53 (2007).

[17] L. P. Yatsenko, B. W. Shore, and K. Bergmann, Phys. Rev. A 89, 013831 (2014).

[18] I. Novikova, R. L. Walsworth, and Y. Xiao, Laser Photonics Rev. 6, 333 (2012).

[19] L. Li, Y. O. Dudin, and A. Kuzmich, Nature (London) 498, 466 (2013).

[20] G. Heinze, C. Hubrich, and T. Halfmann, Phys. Rev. Lett. 111, 033601 (2013).

[21] J. H. Wesenberg, K. Mølmer, L. Rippe, and S. Kröll, Phys. Rev. A 75, 012304 (2007).

[22] X. Yang, M. Yu, D.-L. Kwong, and C. W. Wong, Phys. Rev. Lett. 102, 173902 (2009).

[23] M. Saffman, T. G. Walker, and K. Mølmer, Rev. Mod. Phys. 82, 2313 (2010).

[24] A. Sargsyan, D. Sarkisyan, and A. Papoyan, Phys. Rev. A 73, 033803 (2006).

[25] M. D. Lukin and A. Imamoglu, Phys. Rev. Lett. 84, 1419 (2000).

[26] D. Petrosyan and G. Kurizki, Phys. Rev. A 65, 033833 (2002).

[27] J. Evers and C. H. Keitel, Europhys. Lett. 68, 370 (2004).

[28] J. Harden, A. Joshi, and J. D. Serna, Eur. J. Phys. 32, 541 (2011).

[29] S. Huang, J. Phys. B 47, 055504 (2014).

[30] S. Li, X. Yang, X. Cao, C. Zhang, C. Xie, and H. Wang, Phys. Rev. Lett. 101, 073602 (2008).
[31] F. E. Zimmer, J. Otterbach, R. G. Unanyan, B. W. Shore, and M. Fleischhauer, Phys. Rev. A 77, 063823 (2008).

[32] A. S. Sheremet, L. V. Gerasimov, I. M. Sokolov, D. V. Kupriyanov, O. S. Mishina, E. Giacobino, and J. Laurat, Phys. Rev. A 82, 033838 (2010).

[33] J. Choi and D. S. Elliott, Phys. Rev. A 89, 013414 (2014).

[34] C. Ottaviani, S. Rebić, D. Vitali, and P. Tombesi, Phys. Rev. A 73, 010301 (2006)

[35] Y. Chen, X. G. Wei, and B. S. Ham, Opt. Express 17, 1781 (2009).

[36] M. Scherman, O. S. Mishina, P. Lombardi, E. Giacobino, and J. Laurat, Opt. Express 20, 4346 (2012).

[37] D. Møller, J. L. Sørensen, J. B. Thomsen, and M. Drewsen, Phys. Rev. A 76, 062321 (2007).

[38] A. Joshi and M. Xiao, Phys. Rev. A 71, 041801 (2005).

[39] J. Klein, F. Beil, and T. Halfmann, Phys. Rev. Lett. 99, 113003 (2007).

[40] G. G. Grigoryan, G. V. Nikoghosyan, T. Halfmann, Y. T. Pashayan-Leroy, C. Leroy, and S. Guerin, Phys. Rev. A 80, 033402 (2009).

[41] Y.-R. Shen, The Principles of Nonlinear Optics (WileyInterscience, New York, 1984), p. 563.

[42] D. Grischkowsky, E. Courtens, and J. A. Armstrong, Phys. Rev. Lett. 31, 422 (1973).

[43] V. Chaltykyan, G. Grigoryan, and G. Nikogosyan, Phys. Rev. A 68, 013819 (2003).

[44] G. G. Grigoryan, Y. T. Pashayan-Leroy, C. Leroy, and S. Guerin, Phys. Rev. A 79, 013813 (2009).

[45] R. Grobe, F. T. Hioe, and J. H. Eberly, Phys. Rev. Lett. 73, 3183 (1994).

[46] P. Lax and B. Wendroff, Commun. Pure Appl. Math. 13, 217 (1960).

[47] L. N. Trefethen, Finite Difference and Spectral Methods for Ordinary and Partial Differential Equations (unpublished), available at http://people.maths.ox.ac.uk/trefethen/pdetext.html. 